\title{
Two-dimensional optics on silicon nitride multilayer: Refraction of Bloch surface waves
}

\author{
Tristan Sfez, ${ }^{1, a)}$ Emiliano Descrovi, ${ }^{2}$ Libo Yu, ${ }^{1}$ Marzia Quaglio, ${ }^{2}$ Lorenzo Dominici, ${ }^{3}$ \\ Wataru Nakagawa, ${ }^{4}$ Francesco Michelotti, ${ }^{3}$ Fabrizio Giorgis, ${ }^{2}$ and Hans Peter Herzig ${ }^{1}$ \\ ${ }^{1}$ Optics and Photonics Technology Laboratory, Ecole Polytechnique Fédérale de Lausanne, EPFL-IMT-OPT, \\ Rue A.-L. Breguet 2, 2000 Neuchâtel, Switzerland \\ ${ }^{2}$ Materials and Microsystems Laboratory \Lab, Politecnico di Torino, C.so Duca degli Abruzzi 24, \\ 10129 Torino, Italy \\ ${ }^{3}$ Dipartimento di Energetica, SAPIENZA, Università di Roma and CNISM, via A. Scarpa 16, \\ 00161 Roma, Italy \\ ${ }^{4}$ Department of Electrical and Computer Engineering, Montana State University, P.O. Box 173780, \\ Bozeman, Montana 59717-3780, USA
}

(Received 24 November 2009; accepted 18 February 2010; published online 12 April 2010)

\begin{abstract}
When properly designed, a dielectric multilayer can sustain Bloch surface waves (BSWs). Using a multiheterodyne scanning near-field optical microscope that resolves phase and polarization, we will show that a thin dielectric structure deposited on the multilayer deflects the BSW propagation according to Snell's law. Moreover, the mechanism involved in this process is a transfer of energy from the BSW state in the bare multilayer to the new BSW state generated by the presence of the thin dielectric structure. No relevant radiative counterpart occurs. This characteristic validates the treatment of BSWs at the surface of dielectric multilayers as a two-dimensional phenomenon. (C) 2010 American Institute of Physics. [doi:10.1063/1.3385729]
\end{abstract}

Bloch surface waves (BSW) are optical surface modes that may appear within the photonic band gap of a truncated, periodic dielectric multilayer. ${ }^{1-3}$ For a carefully designed multilayer, such BSWs manifest certain similarities with surface plasmon polaritons (SPPs). Mainly, the optical field is highly confined close to the interface with the outer medium. ${ }^{4}$ As SPPs, this characteristic leads to BSW sensing applications. ${ }^{5-7}$ The surface confinement suggests that it may be useful to describe the propagation of BSWs with simple two-dimensional (2D) laws. Such downscaling of a threedimensional model to a 2D one is not only of fundamental interest, but could facilitate the development of devices based on these structures.

SPP propagation through different kinds of interfaces was already extensively studied. Griesing et $\mathrm{al}^{8}{ }^{8}$ studied the propagation through dielectric microprisms deposited on a metal layer. Zhang et al. ${ }^{9}$ looked at propagation through convex and concave lens-type dielectric structures laying on a metal layer. Kitazawa et al. ${ }^{10}$ observed SPPs sustained at the interface between these $\mathrm{Al}$ and $\mathrm{Au}$ layers. These experiments confirm that the transmission of the SPP through such interfaces manifests a refractive-type behavior governed by Snell's law.

BSWs possess specific properties that differentiate them from SPPs. Sustained by a dielectric structure, they do not suffer from the losses due to metals also. The mode of a BSW extends into the multilayer more than the evanescent tail of the SPPs in the metallic layer(s). We report here on the use of a multiheterodyne scanning near-field optical microscope (MH-SNOM) to perform polarization- and phaseresolved mapping of the propagating BSWs modes. The experimental results show that despite the differences between SPPs and BSWs, Snell's law still accurately describes the

${ }^{a)}$ Electronic mail: tristan.sfez@epfl.ch. propagation of BSWs through thin dielectric structures.

The sample used in the MH-SNOM experiment is a hydrogen doped amorphous silicon nitride multilayer grown by plasma enhanced chemical vapor deposition (PECVD) on a glass substrate. The refractive index of the layers is tuned by controlling the concentration of ammonia in the PECVD process. The multilayer is composed of ten periods of alternating high index $\left(\mathrm{n}_{h}=2.23\right.$ at $\left.\lambda=1530 \mathrm{~nm}\right)$ and low index $\left(\mathrm{n}_{l}=1.75\right.$ at $\left.\lambda=1530 \mathrm{~nm}\right)$ layers whose thicknesses are $\mathrm{d}_{l}$ $=294 \mathrm{~nm}$ and $\mathrm{d}_{h}=240 \mathrm{~nm}$, respectively. The multilayer was designed to support only TE-polarized BSW. ${ }^{12}$ Polymeric ridges (width $\mathrm{w}=10 \mu \mathrm{m}$, height $\mathrm{h}=110 \mathrm{~nm}$ ) were then deposited on top of the multilayer. The polymer (AZ5214E, Clariant $\mathrm{GmbH})$ is used as a positive photoresist $\left(\mathrm{n}_{\text {pol }}\right.$ $=1.66$ ) for photolithography. Fabrication details of the ridges and the multilayer may be found in Refs. 11 and 12, respectively.

A schematic view of the structure is shown in Fig. 1(a). Figure 1(b) presents the measured far-field dispersion curves of the bare multilayer (left) and the multilayer with an additional $110 \mathrm{~nm}$ thick polymer film (right). BSWs are excited with TE-polarized incident light. The air-glass line is indicated by the vertical line. The additional polymer layer shifts the resonance to about $100 \mathrm{~nm}$ longer wavelengths for a fixed incident angle. The far-field measurement method is described in Ref. 12

The MH-SNOM (see Refs. 12-15 for a detailed description) is used to investigate the optical response of the structure. The BSWs are excited in the Kretschmann configuration with a BK7 prism $\left(\mathrm{n}_{p}=1.50\right)$. The SNOM probe (Lovalite, $200 \mathrm{~nm}$ aperture, $70 \mathrm{~nm} \mathrm{Al}$ coated) collects the optical near field at the surface of the sample. In addition, the shear-force feedback (APE Research system) provides the topography. The MH-SNOM allows the simultaneous detection of the amplitude and phase of the optical near fields generated by two orthogonal states of polarization compos- 


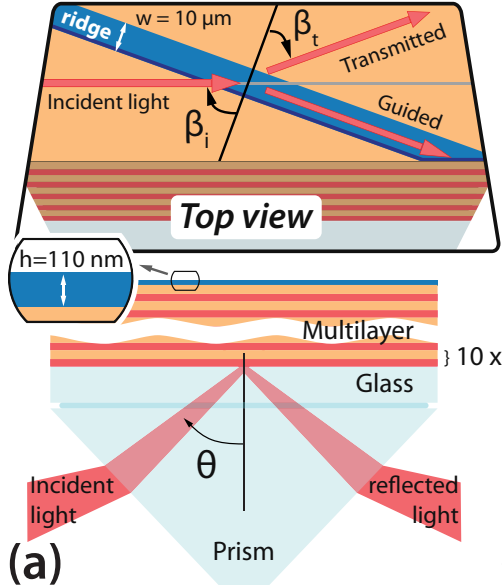

(b)

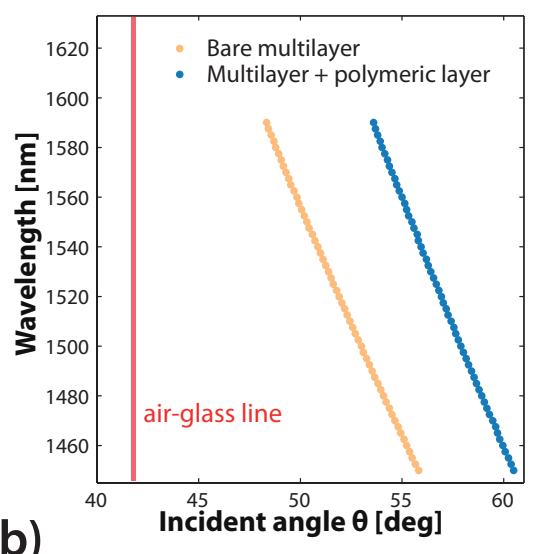

FIG. 1. (Color online) (a) The sample consists of a thin polymer ridge $($ width $=10 \mu \mathrm{m}$, height $=110 \mathrm{~nm})$ deposited on a silicon nitride multilayer. (b) Measured dispersion curves for the bare multilayer (left) and for the multilayer with an additional $110 \mathrm{~nm}$ polymer layer deposited on top (right). ing the illuminating beam, TE and TM in our experiment. The incident light is shaped with a focuser whose divergence is $4^{\circ}$ with a spot size of about $20 \mu \mathrm{m}$ at the surface of the sample. The focuser is mounted on a goniometer which allows to change the coupling angle $\theta$.

Figures 2(a)-2(c), respectively, show the topography, the TM- and TE- polarized near fields as simultaneously obtained from the MH-SNOM $(\theta=57.2 \mathrm{deg}, \lambda=1555 \mathrm{~nm})$. According to the dispersion curves in Fig. 1(b) and the divergence of the illuminating beam, the BSW can only be excited in the polymer coated multilayer. The incident light arrives from the left and its projection onto the sample surface makes an angle $\beta_{i}$ of $65^{\circ}$ with respect to the normal to the ridge. The dashed lines mark the edges of the ridge as obtained from the topography. The Airy pattern of the TM beam delimitates the incidence region. The TE-polarized light excites a BSW in the polymeric ridge and then leaks out at $\beta_{t}=76^{\circ}$, i.e., $14^{\circ}$ with respect to the ridge. One can also find an equivalent angular contribution at $14^{\circ}$ at the bottom of the ridge. It corresponds to that part of the BSW which is reflected at the upper interface and transmitted at the lower interface. In addition, by increasing $\beta_{i}$, we reach the critical angle $\beta_{\mathrm{TIR}}$ above which the BSW is trapped and guided in the ridge through total internal reflection (TIR). This situation is showed in Fig. 2(d) where $\beta_{i}=75^{\circ}$. Guiding of BSWs have already been reported in Ref. 11 .
A series of measurements is then performed at different $\beta_{i}\left(\beta_{i}<\beta_{\text {TIR }}, \lambda=1580 \mathrm{~nm}\right)$. The red diamonds in Fig. 2(e) are the experimental values: $\sin \beta_{t}$ is plotted as a function of $\sin \beta_{i}$. The phase matching condition expressing the BSW coupling from the polymer coated multilayer to the bare multilayer is

$$
n_{\mathrm{BSW}}^{b} \cdot \sin \beta_{t}=n_{\mathrm{BSW}}^{c} \cdot \sin \beta_{i},
$$

where $n_{\mathrm{BSW}}^{b}$ and $n_{\mathrm{BSW}}^{c}$ are the BSW effective indices in the bare and coated multilayer, respectively. They can be deduced from the far-field measurements through $n_{\mathrm{BSW}}^{b}$ $=n_{p} \sin \theta_{b}$ and $n_{\mathrm{BSW}}^{c}=n_{p} \sin \theta_{c}$, where $\theta_{r}$ and $\theta_{b}$ are the coupling incident angles in the bare and coated multilayer, respectively. Using the values $\theta_{b}=48.8^{\circ}$ and $\theta_{c}=54.0^{\circ}$ obtained at $\lambda=1580 \mathrm{~nm}$ from Fig. 1(b), the Eq. (1) gives the solid line (blue on-line) plotted in Fig. 2(e), along with the equivalent for coupling from the polymer coated multilayer to air (in black). The linear fitting of the experimental data provides a slope of $1.068\left(R^{2} \simeq 0.99\right)$, which corresponds to a discrepancy of less than $0.8 \%$ with respect to the slope of 1.076 given by Eq. (1).

As the width of the ridge is more than seven times the effective wavelength, in a simple model, we can assume that the $10 \mu \mathrm{m}$ wide ridge behaves as a semi-infinite layer. ${ }^{16}$ The observed phenomenon is hence a refraction of BSWs at an interface between two effective media as follows: a bare di-
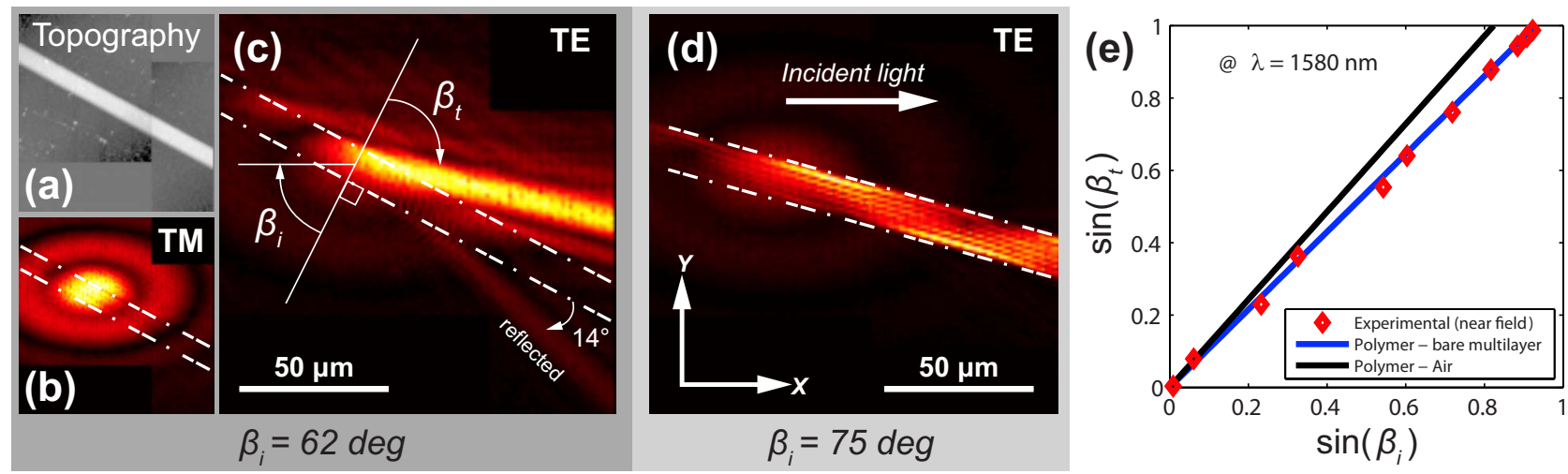

FIG. 2. (Color online) The simultaneously measured (a) topography, (b) TM- and (c) TE-polarized near fields. The dashed lines correspond to the edges of the ridge. In the plane of the sample, the incident light arrives from the left and makes an angle $\beta_{i}$ of $62^{\circ}$ with respect to the ridge normal. The TE-light goes out of the polymeric ridge at a transmitted angle of $\beta_{t}$ of $76^{\circ}$. (d) The incident angle $\beta_{i}=75^{\circ}$ exceeds the critical angle and the TE-polarized field is trapped in the ridge $(\lambda=1555 \mathrm{~nm})$. (e) Measured $\sin \left(\beta_{t}\right)$ for several values of $\sin \left(\beta_{i}\right)$ (diamonds). The lower and upper straight lines represent the calculated refraction angles expected at a polymer-bare multilayer and polymer-air interface, respectively $(\lambda=1580 \mathrm{~nm})$. 

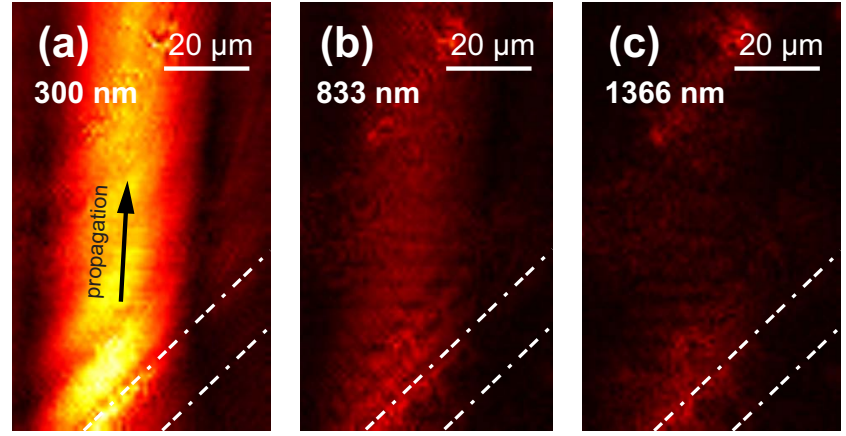

FIG. 3. (Color online) TE-polarized MH-SNOM amplitudes at different heights above the sample surface: (a) 300, (b) 833, and (c) $1366 \mathrm{~nm}$. The dashed lines are the edges of the ridge.

electric multilayer and the polymer coated multilayer. The refractive behavior is well described by Snell's law.

Because the probe collects the near field, there may be an unseen radiative component accompanying this phenomenon, i.e., some diffracted light corresponding to a transmission from the BSW in the coated multilayer to the air. In order to clarify this point, we turned off the shear-force feedback and make a series of scans at different heights above the sample surface. Figures 3(a)-3(c) corresponds to the measurements taken, respectively, at $300 \mathrm{~nm}, 833 \mathrm{~nm}$ and $1366 \mathrm{~nm}$ above the surface. In these maps, the BSW propagates from the bottom to the top. As can be seen, no other relevant angular contribution appears: at $1366 \mathrm{~nm}$, almost all of the signal intensity vanishes. The signal that is seen close to the ridge (dashed lines) corresponds to light scattered at the edge of the ridge. The scattering that occurs at the top of the figures is due to sample inhomogeneities. We therefore conclude that no significant radiative component exists due to the polymer-air interface.

The observed refractive behavior may be imaged in 2D by looking at the wavefronts on the surface of the sample. We set the incident angle $\theta$ to $53.6^{\circ}$ and the wavelength to $1492 \mathrm{~nm}$, and record the amplitude and phase maps. Under these conditions, the TE-polarized light is coupled to the BSW in the bare multilayer [see Fig. 1(b)]. Figure 4(a) shows the effect of a ridge turned by $\beta_{i}=58.5^{\circ}$ with respect
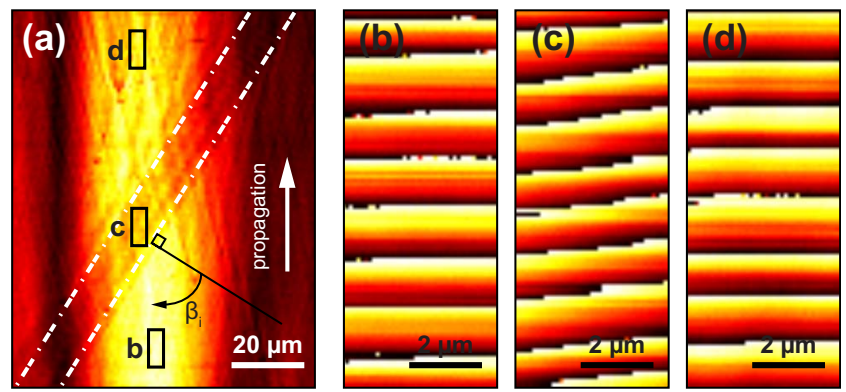

FIG. 4. (Color online) (a) Transmission of the TE-polarized BSW through the ridge (dashed lines). The BSW propagates toward the top of the figure. In (b), (c), and (d) are the phase profile wavefronts corresponding to the black rectangles labeled $b, c$, and $d$ in (a) $(\lambda=1492 \mathrm{~nm})$. to the propagative BSW. After a deflection to the left within the ridge, the BSW goes out of the structure parallel to the initial BSW. As the MH-SNOM is phase sensitive, the phase has been captured in small areas of $5 \times 10 \mu \mathrm{m}$ before, within, and after the ridge: Figs. 4(b)-4(d), respectively. Consistent with the vertical propagation of the BSW before and after the ridge, the wavefronts are flat in (b) and (d). In (c), the wavefronts are tilted by approximately $6 \pm 2.8^{\circ}$ with respect to the horizontal. Using Snell's law (1), the expected angle is $4.9^{\circ}$ [with $\theta_{b}=53.4^{\circ}$ and $\theta_{c}=58.3^{\circ}$ from Fig. 1(b)], which agrees reasonably well with the measured value. These results indicate that the in-plane orientation of the wave vectors across the interfaces satisfies the phase matching condition. As in classical textbooks, the latter assertion leads to Snell's law.

In conclusion, we applied the MH-SNOM to perform a polarization and phase-resolved characterization of the BSW propagative modes in a multilayer dielectric structure. These results demonstrate that the only mechanism responsible for the transmission of a BSW through a thin dielectric relief is an energy transfer from the BSW mode of the bare multilayer to the BSW mode of the coated multilayer. At the interface, the deflection of the propagation direction is well described by the 2D Snell's law. A familiar law can therefore be used for the conception of thin optical dielectric structures involving BSWs.

This work was supported by the Swiss National Science Foundation, by the Piedmont Regional project CIPE 2008 "Photonic biOsensors for Early caNcer dIagnostics (PHOENICS)" and the Science and Technology Atheneum Research Programme of the SAPIENZA University.

${ }^{1}$ P. Yeh, A. Yariv, and A. Y. Cho, Appl. Phys. Lett. 32, 104 (1978).

${ }^{2}$ W. M. Robertson, G. Arjavalingam, R. D. Meade, K. D. Brommer, A. M. Rappe, and J. D. Joannopoulos, Opt. Lett. 18, 528 (1993).

${ }^{3}$ J. Gaspar-Armenta and F. Villa, J. Opt. Soc. Am. B 21, 405 (2004).

${ }^{4}$ C. Vandenbem, Opt. Lett. 33, 2260 (2008).

${ }^{5}$ E. Guillermain, V. Lysenko, R. Orobtchouk, T. Benyattou, S. Roux, A. Pillonnet, and P. Perriat, Appl. Phys. Lett. 90, 241116 (2007).

${ }^{6}$ E. Descrovi, F. Frascella, B. Sciacca, F. Geobaldo, L. Dominici, and F. Michelotti, Appl. Phys. Lett. 91, 241109 (2007).

${ }^{7}$ M. Liscidini and J. E. Sipe, Appl. Phys. Lett. 91, 253125 (2007).

${ }^{8}$ S. Griesing, A. Englisch, and U. Hartmann, Opt. Lett. 33, 575 (2008).

${ }^{9}$ D. G. Zhang, X. C. Yuan, J. Bu, G. H. Yuan, Q. Wang, J. Lin, X. J. Zhang, P. Wang, H. Ming, and T. Mei, Opt. Express 17, 11315 (2009).

${ }^{10}$ T. Kitazawa, S. Miyanishi, Y. Murakami, K. Kojima, and A. Takahashi, Phys. Rev. B 77, 193404 (2008).

${ }^{11}$ E. Descrovi, T. Sfez, M. Quaglio, D. Brunazzo, L. Dominici, F. Michelotti, H.-P. Herzig, O. J. F. Martin, and F. Giorgis, "Guided Bloch surface waves on ultra-thin polymeric ridges" (unpublished).

${ }^{12}$ E. Descrovi, T. Sfez, L. Dominici, W. Nakagawa, F. Michelotti, F. Giorgis, and H. P. Herzig, Opt. Express 16, 5453 (2008).

${ }^{13}$ R. Dändliker, P. Tortora, L. Vaccaro, and A. Nesci, J. Opt. A, Pure Appl. Opt. 6, S18 (2004).

${ }^{14}$ B. Bai, X. Meng, J. Laukkanen, T. Sfez, L. Yu, W. Nakagawa, H.-P Herzig, L. Li, and J. Turunen, Phys. Rev. B 80, 035407 (2009).

${ }^{15}$ T. Sfez, E. Descrovi, L. Dominici, W. Nakagawa, F. Michelotti, F. Giorgis, and H.-P. Herzig, Appl. Phys. Lett. 93, 061108 (2008).

${ }^{16}$ T. Holmgaard and S. I. Bozhevolnyi, Phys. Rev. B 75, 245405 (2007). 